\title{
VIDEO-ASSISTED CARDIOSCOPIC RESECTION OF RECURRENT LEFT VENTRICULAR MYXOMA
}

\author{
Jiun-Yi Li, MD, Fang-Yue Lin, MD, Ron-Bin Hsu, MD, and Shu-Hsun Chu, MD, Taipei, Taiwan, Republic of China
}

Cardiac myxoma most often arises from the left atrium, where $80 \%$ to $90 \%$ of cardiac myxomas are located. Ventricular myxomas comprise fewer than $5 \%$ of all cardiac myxomas. Those located in the left ventricle are extremely rare. Traditionally, removal of a left ventricular myxoma is done through aortotomy if the myxoma is located at the left ventricular outflow tract and through the atrium for other locations. We report a case of recurrent deep-seated left ventricular myxoma removed through an aortotomy with video-assisted cardioscopy.

Case report. A 20-year-old woman had a first episode of near-syncope 3 years before the operation under discussion was done. Transient bilateral visual loss, general weakness, palpitation, and cold sweating were noted. She had full recovery from the symptoms after squatting for 10 minutes. Since then, she had exercise intolerance and exertional chest pain. Episodes of transient visual loss occurred frequently. One year after the first episode she had a grade 4 systolic ejection murmur over the left upper sternal border with radiation to the aortic area. A midsystolic plop was audible over the cardiac apical area. A thrill was palpable with some heartbeats in the aortic area. No signs of congestive heart failure were noted. Echocardiography showed a $6 \times 5 \mathrm{~cm}^{2}$ tumor originating from the interventricular septum near the posterior papillary muscle. The tumor compressed the left ventricular outflow tract during systole and caused mild mitral prolapse. Cardiac catheterization confirmed the presence of the tumor at the inflow tract of the left ventricle.

An emergency operation had been undertaken at that time. With the use of cardiopulmonary bypass and cardioplegic protection, the left atrium was approached through an interatrial incision. Because of poor visualization of the tumor through the mitral valve, the anterior mitral leaflet was detached. A $6 \times 5 \times 5 \mathrm{~cm}^{3}$ multilobulated grayish-brown tumor was found in the left ventricle with a stalk from the interventricular septum. The entire tumor was removed piece by piece. However, the underlying endocardium was not resected. On pathologic examination the tumor was composed of round, polygonal, or stellate cells surrounded by abundant eosinophilic myxoid substance, which is typical of a cardiac myxoma. She had

From the Departments of Surgery, National Taiwan University Hospital, Taipei, Taiwan, Republic of China.

Received for publication April 23, 1996; accepted for publication May 8, 1996.

Address for reprints: Fang-Yue Lin, MD, Department of Surgery, National Taiwan University Hospital, No. 7, ChungShan S. Rd., Taipei, Taiwan, Republic of China.

J Thorac Cardiovase Surg 1996;112:1673-4

Copyright (C) 1996 by Mosby-Year Book, Inc.

$0022-5223 / 96 \$ 5.00+0 \quad \mathbf{1 2 / 5 4 / 7 4 9 0 7}$ an uneventful postoperative course and no residual tumor was found by echocardiography.

She was regularly followed up in the outpatient clinic. No discomfort was noted after the operation. However, a $2.1 \times 1.9 \mathrm{~cm}^{2}$ tumor with a stalk attached to the interventricular septum was demonstrated by echocardiography 2 years after the first operation. The tumor was thought to be a recurrent left ventricular myxoma.

A repeat operation was done. Using cardiopulmonary bypass, a transverse aortotomy was done. The cardioscope was inserted and a multilobulated jellylike tumor at the base of the anterior septum near the posterior papillary muscle was noted (Fig. 1). The tumor with a piece of myocardium beneath it was removed piece by piece under video-assisted cardioscopic visualization. The tumor was gelatinous, transparent, and soft. Microscopically, it was shown to be a myxoma composed of stellate cells in myxoid stroma.

The patient had an uneventful recovery after the second operation.

Discussion. Left ventricular myxomas are extremely rare. Only 16 cases had been reported in the English literature up to $1993 .{ }^{1}$ The duration of clinical symptoms caused by left ventricular myxoma was no longer than 4 years. ${ }^{2}$ It was suggested that the growth rate of the tumor was different from that of myxomas at other locations. However, clinical symptoms were not a reliable indicator because they were related to several additional factors, including the precise location of the tumor. Recurrence of myxomas at other locations was reported to occur 17 years on the average after initial presentation. ${ }^{1}$ This case is the first reported of a recurrent isolated left ventricular

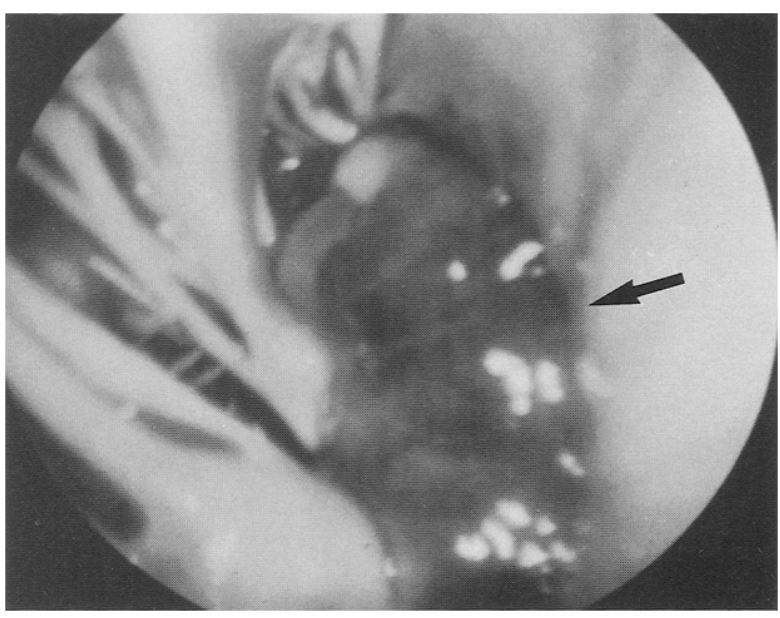

Fig. 1. Photograph of myxoma under transaortotomy cardioscopy. Tumor (arrow) is located at interventricular septum near base of posterior papillary muscle. 


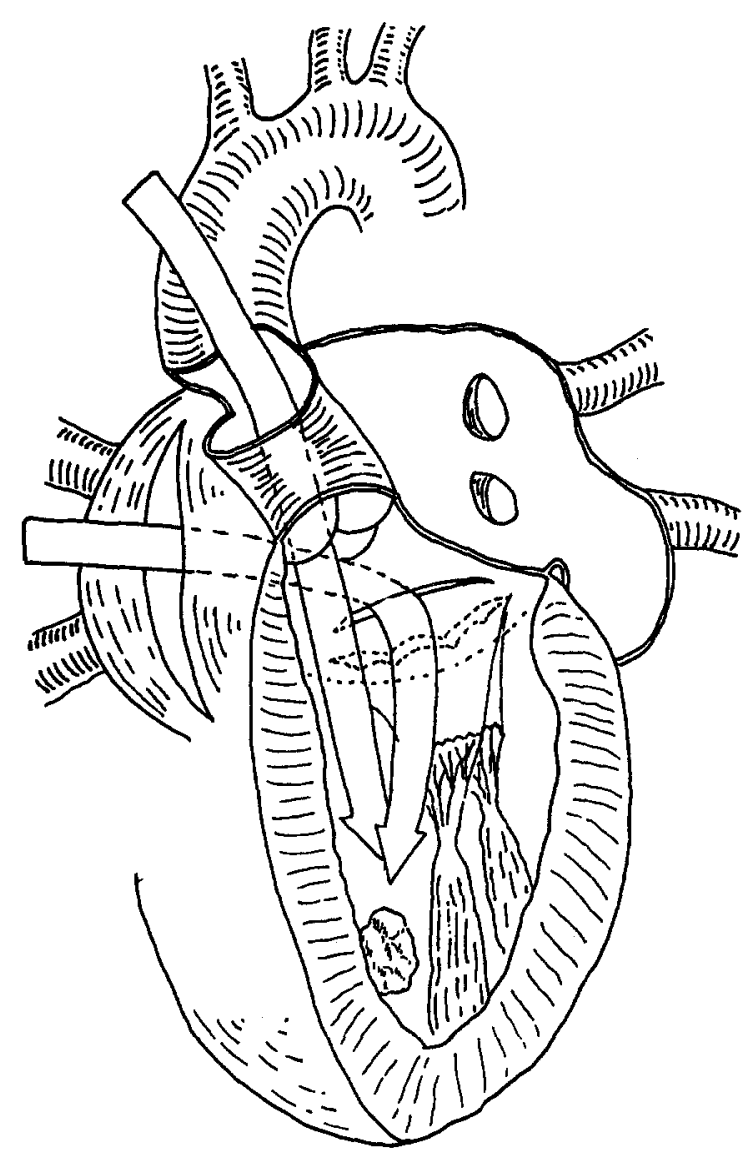

Fig. 2. Approach through left atrium is difficult because angle (curved arrow) exists between mitral anulus and tumor. Transaortotomy approach provides better visualization and complete removal of tumor (straight arrow).

myxoma. If the original myxoma was resected incompletely because the underlying myocardium was not resected and the recurrent tumor was found to be $2 \mathrm{~cm}$ in size 2 years later, the estimated doubling time of the tumor was 1 to 2 months.

The cause of recurrence might have been incomplete resection inside the endocardium or a new growth, especially in view of the reports that some myxomas are infiltrative. The unusual location of the myxoma in the left ventricle made complete resection difficult and possibly lethal. ${ }^{3}$ Multigrowth potential of the tumor has also been mentioned and was thought to be an important factor. ${ }^{1}$ Although no residual tumor was noted at follow-up echocardiography, infiltration of the myxoma into the myocardium is highly possible.

In a previous report, left ventricular myxomas in the inflow tract could be removed through a left atrial approach whereas those in the outflow tract were removed through an aortotomy. ${ }^{4}$ The previous method was applied during the first operation in the present case. The tumor was so difficult to approach that we detached the anterior leaflet of the mitral valve. The completeness of resection was limited because of the difficulty in adequate visualization of the entire left ventricular cavity. During the second operation, the location of the tumor on the interventricular septum near the posterior papillary muscle and the small size of the tumor made an approach through the left atrium and the mitral valve even more difficult. Videoassisted cardioscopy through an aortotomy provided good visualization and magnification of the intraventricular structures and of the location of the tumor (Fig. 2). The relationship between the tumor and the papillary muscle and the interventricular septum could be identified. After resection of the tumor, the entire left ventricular cavity could be inspected thoroughly. Video-assisted cardioscopy has been reported in difficult operations for congenital lesions of the heart to avoid vigorous cardiac manipulation and an extended incision. ${ }^{5}$ We applied the technique in this patient for the removal of a deep-seated left ventricular myxoma. A left atriotomy and detachment of the mitral leaflet could be avoided and the operative result was satisfactory.

We conclude that video-assisted cardioscopy provides an excellent view of a deep-seated left ventricular tumor and aids in its resection.

\section{REFERENCES}

1. Castell E, Ferran V, Octavio de Toledo MC, et al. Cardiac myxomas: surgical treatment, long-term results and recurrence. J Cardiovasc Surg 1993;34:49-53.

2. Rosenzweig A, Harrigan P, Popvic AD. Left ventricular myxoma simulating aortic stenosis. Am Heart J 1989;117: 962-3.

3. Landau E, Reisin LH, LV myxoma resembling restrictive cardiomyopathy [letter]. Am Heart J 1986;112:1356.

4. Kirklin JW, Barratt-Boyes BG. Cardiac tumor. In: Cardiac surgery. 2nd ed. New York: Churchill Livingstone, 1993:163553.

5. Burke RP, Michielon G, Wernovsky G. Video-assisted cardioscopy in congenital heart operations. Ann Thorac Surg 1994;58:864-8. 